\title{
Inactivation of AKT, ERK and NF-kB by genistein derivative, 7-difluoromethoxyl-5,4'-di-n-octylygenistein, reduces ovarian carcinoma oncogenicity
}

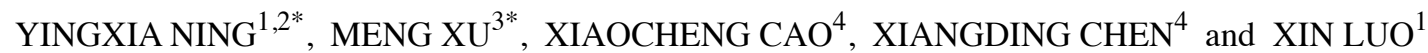

${ }^{1}$ Department of Gynecology and Obstetrics, The First Affiliated Hospital of Jinan University, Guangzhou, Guangdong 510630;

${ }^{2}$ Department of Gynecology and Obstetrics, The First Affiliated Hospital of Guangzhou Medical University,

Guangzhou, Guangdong 510120; ${ }^{3}$ Department of Oncology, The First Affiliated Hospital of Jinan University, Guangzhou, Guangdong 510120; ${ }^{4}$ Laboratory of Molecular and Statistical Genetics,

Hunan Normal University, Changsha, Hunan 410081, P.R. China

Received January 19, 2017; Accepted March 3, 2017

DOI: $10.3892 /$ or.2017.5709

\begin{abstract}
Cancer stem cells (CSCs) have central functions in cancer formation and development. Aberrant expression of AKT, ERK and NF- $\kappa \mathrm{B}$ signaling pathways have been reported in several types of CSCs. Phytochemicals from dietary compounds possess anti-CSC properties, and have been characterized as promising therapeutic agents for the prevention and treatment of many types of cancers. We previously showed that the newly synthesized genistein derivative, 7-difluoromethoxyl-5,4'-di-n-octylygenistein (DFOG), can inhibit the self-renewal ability of ovarian cancer stem cells (OVCSLCs). In the present study, we further assessed whether various signaling pathways are regulated by DFOG. We found that spheroids derived from the SKOV3 cell line possessed OVCSLC properties and DFOG efficiently inhibited the stemness of the OVCSLCs. In addition, the suppression of spheroid and colony formation by DFOG was associated with inhibition of AKT and ERK $1 / 2$ protein phosphorylation, and NF- $\mathrm{KB}$ activity in OVCSLCs from the SKOV3 cells. Importantly, DFOG inhibited the oncogenicity of the OVCSLCs by activation of FoxO3a and/or inactivation of FoxM1 by the targeting of multiple pro-survival (AKT and ERK1/2) and proinflammatory $(\mathrm{NF}-\mathrm{\kappa B})$ pathways, providing a new avenue for the treatment of ovarian carcinoma in humans.
\end{abstract}

Correspondence to: Dr Xin Luo, Department of Gynecology and Obstetrics, The First Affiliated Hospital of Jinan University, 613 Huangpu West Road, Guangzhou, Guangdong 510630, P.R. China

E-mail: luoxin2016@yeah.net

*Contributed equally

Key words: 7-difluoromethoxyl-5,4'-di-n-octylygenistein, ovarian cancer stem-like cells, Akt, ERK1/2, NF-кB, FoxO3a, FoxM1, carcinogenicity

\section{Introduction}

Several signaling pathways are crucial in orchestrating cancer stem cell (CSC) activity. Dysregulation of these pathways has been implicated in the maintenance and function of CSCs. A study by Sunayama et al showed that simultaneous blocking of phosphoinositide 3-kinase (PI3K)/AKT and extracellular signal-regulated kinase (ERK1/2) signaling promoted glioblastoma cancer stem-like cell (CSLC) differentiation, suppressing tumorigenicity by activating FoxO3a, which is commonly phosphorylated by AKT and ERK for signal transduction (1). Jacobsen et al reported that deregulation of Foxo3a and NF- $\kappa \mathrm{B} / \mathrm{Rel}$ is associated with malignancy in SCID temperature-sensitive form of Abelson mouse leukemia virus pre-B cells (2). Our previous studies demonstrated that FoxO3a inactivation and/or FoxM1 activation are essential for oncogenicity as well as stemness in ovarian cancer stem-like cells (OVCSLCs) derived from established ovarian cancer cell lines $(3,4)$. However, whether FoxO3a can integrate these pro-survival and pro-inflammatory pathways to induce tumorigenicity in OVCSLCs remains to be investigated.

Numerous epidemiological studies have substantiated the efficient anticancer properties of dietary components in vegetables and fruits (5). These bioactive and non/low-toxic phytochemicals are considered promising candidates for cancer intervention (5,6). Genistein, 5,7,4'-trihydroxylisoflavone, a major soybean compound, possesses antitumor properties $(7,8)$. However, genistein has poor bioavailability due to its low solubility in both organic solvents and water (9). Notably, the introduction of $\mathrm{HCF}_{2}$ or $\mathrm{CF}_{3}$ into the genistein molecule improves the anticancer activities of genistein derivatives (9). Furthermore, the newly synthesized genistein derivative, 7-difluoromethoxyl-5,4'-di-n-octylygenistein (DFOG), was found to induce apoptosis in ovarian and gastric carcinomas $(3,4)$. DFOG acts as an inhibitor of CSCs or tumor-initiating cells by the activation of FoxO3a and/or inactivation of FoxM1 $(3,4)$. Nevertheless, whether DFOG inhibits oncogenicity in OVCSLCs by activating FoxO3a and/or inactivating FoxM1 through targeting of multiple pro-survival 
(AKT and ERK1/2) and pro-inflammatory (NF-kB) pathways is unclear.

In the present study, we demonstrated that DFOG suppressed in vitro spheroid and colony formation on soft agar of OVCSLCs obtained from SKOV3 cells. Mechanistically, DFOG exhibited effects similar to those of the PI3K inhibitor (LY294002), MEK inhibitor (U0126) and NF-kB inhibitor (PDTC), simultaneously. The suppressive activity of DFOG on spheroid formation in serum-free medium (SFM) and colony formation on soft agar were dependent on FoxO3a and FoxM1 protein levels. These results indicate that DFOG may be potentially used for the treatment of human ovarian cancer.

\section{Materials and methods}

Reagents. Invitrogen Life Technologies (Shanghai, China) supplied Dulbecco's modified Eagle's medium (DMEM) and DMEM/F12, trypsin-EDTA, fetal bovine serum (FBS) and penicillin-streptomycin. Monoclonal antibodies raised in mice against human anti- $\beta$-actin were manufactured by Sigma-Aldrich (St. Louis, MO, USA) (catalog no. A2066). Rabbit polyclonal antibodies targeting CD44, ALDH1, CD133 and FoxO3a were obtained from Abcam Co. (Cambridge, MA, USA) (catalog nos. ab24504, ab9883, ab19898 and ab53287, respectively). Monoclonal antibodies against FoxM1 (C-20) and NF- $\kappa$ Bp 65 raised in rabbits were manufactured by Santa Cruz Biotechnology, Inc. (Beverly, MA, USA) (catalog nos. sc-502 and sc-8008). Primary antibodies against phospho-AKT (Ser473), AKT, p-ERK (Thr202/Tyr204), phospho-FoxO3a (Ser253) and ERK1/2 were manufactured by Cell Signaling Technology (Danvers, MA, USA) (catalog nos. \#9171, \#9272, \#9101, \#9466 and \#9102).

The pHBad-U6-GFP, pHBad-U6-GFP-shFOXO3a and pHBad-U6-GFP-shFOXM1 plasmid packaging adenoviral particles were obtained from Hanbio Biotechnology Co. Ltd. (Shanghai, China) $\left(2.0 \mathrm{ml}, 1 \times 10^{11} \mathrm{PFU} / \mathrm{ml}\right)$. Pyrrolidine dithiocarbamate, ammonium salt (PDTC), LY294002 and U0126 were purchased from Sigma-Aldrich.

Cell culture and sphere formation assay. Human ovarian carcinoma SKOV3 cells were obtained from the Chinese Academy of Sciences (Shanghai, China) and cultured in DMEM containing 10\% FBS.

Sphere formation was assessed in serum-free culture medium containing antibiotics, growth factors, vitamin B27, and the N2 supplement (Invitrogen), following instructions from the manufacturer. Cell seeding was performed at $10^{4}$ cells/well in 6-well ultra-low attachment plates (Corning, Corning, NY, USA).

Spheroids were obtained by centrifugation $(200 \mathrm{x} \mathrm{g})$ and trypsin-EDTA digestion, followed by mechanical disruption. Single cells were washed and transferred into SFM for sphere induction. Second-generation spheroids were used as ovarian cancer stem-like cells (OVCSLCs).

Single cells with potential for transformation into new spheroids were cultured at 1,000 cells/well in a 24 -well plate, to generate new spheroids. Tumor spheroids were counted in 6 day cultures; the efficiency of spheroid formation was expressed as the ratio of the total number of spheroids generated to that of SKOV3 cells seeded, multiplied by 100 .
Colony formation assay. In the present study, soft agar was used. Medium containing $0.7 \%$ agarose was added into a 6-well plate. Then, $10^{4}$ cells were seeded/well in medium containing $0.4 \%$ agarose (top layer), and incubated for 3 weeks. Routine colony count was carried out on an inverted microscope (Olympus IX53; Olympus, Tokyo, Japan). Three independent experiments were carried out.

In vivo tumorigenicity experiments. Balb/c-nu mice aged 4 weeks were purchased from the Animal Institute of the Chinese Academy of Medical Science (CAMS). All animal studies were performed in accordance with the standard protocols approved by the Ethics Committee of The First Affiliated Hospital of Jinan University and the Committee of Experimental Animal Feeding and Management. Mice were randomly divided into 3 groups (4 mice/group) and maintained under standard conditions, according to the standard protocols. Cells were suspended in serum free-DMEM/Matrigel (BD Biosciences) mixture (1:1 volume). Each recipient Balb/c-nu mouse was inoculated subcutaneously with various numbers of SKOV3-derived OVCSLCs $\left(1 \times 10^{3}, 1 \times 10^{4}\right.$ and $1 \times 10^{5}$ cells) in one flank and the monolayer SKOV3 cells $\left(1 \times 10^{4}, 1 \times 10^{5}\right.$ and $\left.1 \times 10^{6}\right)$ in the other, respectively. Tumorigenicity experiments were terminated 1 month after cell inoculation. Harvested tumors were imaged and weighed immediately. After that, specimens from tumor tissue samples were fixed in $10 \%$ neutral buffered formalin, processed in paraffin blocks, and sectioned. The sections were stained with $\mathrm{H} \& \mathrm{E}$ and examined for the histopathology.

Transduction of shFOXO3a and shFOXM1. SKOV3 cell-derived OVCSLCs were plated into 24-well culture plates at $40-50 \%$ confluency, and incubated overnight. Then, the cells were transduced with the pHBad-U6-GFP or pHBad-U6-GFP-shFOXO3a or pHBad-U6-GFP-shFOXM1 plasmid packaging adenoviral particles using an enhanced infection solution (ENi.s; cat. no. REVG0002; GeneChem, Shanghai, China). Following $4 \mathrm{~h}$ of transduction, DMEM with $10 \%$ fetal calf serum (FCS) was added to replace the transduction medium; this was followed by $48 \mathrm{~h}$ of incubation before gene and protein level assessments.

Western blot analysis. Cells lysis was performed according to published protocols (10). Monoclonal anti- $\beta$-actin, anti-NF-кBp65, anti-FoxM1, anti-phospho-AKT (Ser473) antibodies, and polyclonal anti-CD44, anti-ALDH1A1, anti-CD133, anti-AKT, anti-p-ERK (Thr202/Tyr204), antiERK1/2, anti-Phospho-FoxO3a (Ser253) antibodies were used as primary antibodies, for overnight incubation at $4^{\circ} \mathrm{C}$. Adequate horseradish peroxidase (HRP) bound secondary antibodies were added for $1 \mathrm{~h}$ at ambient temperature; visualization of specific protein bands was carried out using enhanced chemiluminescence, with $\beta$-actin employed for normalization.

Statistical analysis. Comparisons were conducted by two-tailed Student's t-test. A P-value $<0.05$ indicated statistical significance.

\section{Results}

Spheroids reflect SKOV3 cell-derived OVCSLCs. CSCs are mainly characterized by their capacity to form 3-dimensional 
A

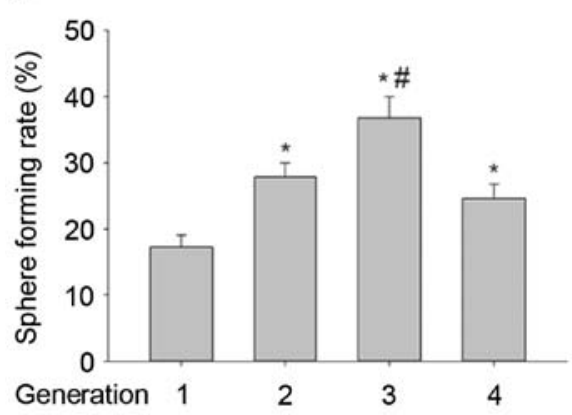

C

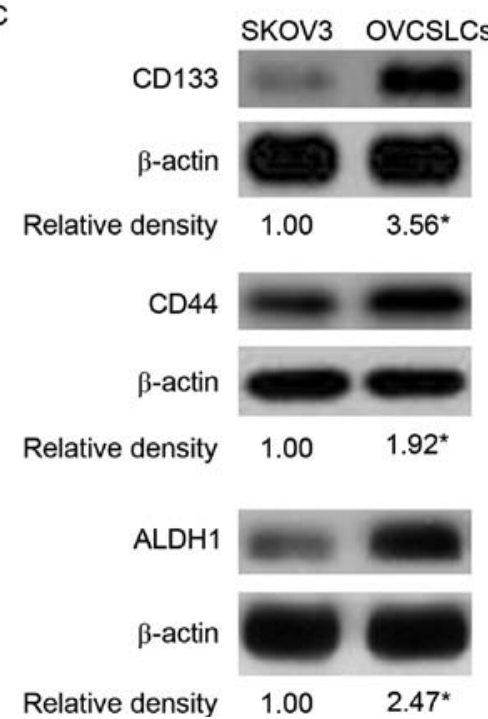

B

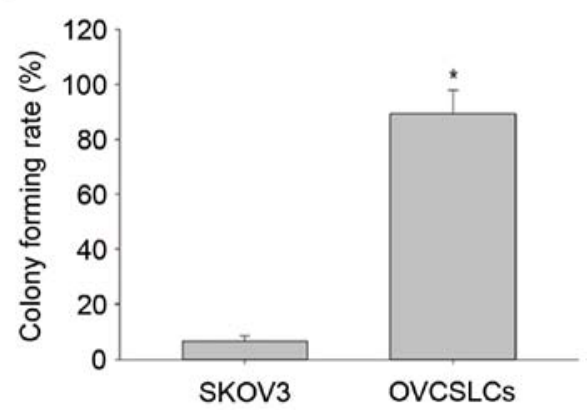

D

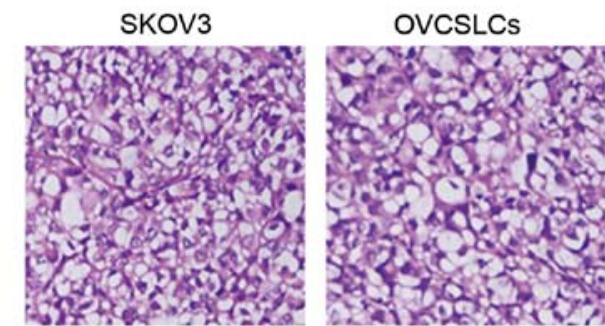

Figure 1. Spheroids display the properties of OVCSLCs in SKOV3 cells. (A) Comparison of spheroid-forming rates in spheroids of different generations ( $\mathrm{P}<0.05$ compared to primary spheroid culture; ${ }^{\text {"P }}<0.05$ compared to second or fourth spheroid culture). (B) Comparison of colony forming rates and (C) protein levels of stem cell markers in OVCSLCs (second-generation spheroids) and SKOV3 cells in monolayer growth (mean \pm SD or mean, ${ }^{*} \mathrm{P}<0.05$ compared to cells in monolayer growth). (D) SKOV3 cell-produced OVCSLCs $\left(10^{4}\right)$ or $10^{5} \mathrm{SKOV} 3$ cells in monolayer growth were subcutaneously injected into male BALB/c-nu mice. After 6 weeks, tumors were obvious in OVCSLC and SKOV3 cell xenotransplantation animals. H\&E staining showed similar histology for xenograft tumors from OVCSLCs and SKOV3 cells. Unless otherwise stated, all experiments were performed in triplicate and values are mean \pm SD.

Table I. Tumorigenicity experiments of OVCSLCs and SKOV3 cells in BALB/c-nu mice.

\begin{tabular}{lccc}
\hline Cells & $\begin{array}{c}\text { Inoculum } \\
\text { amount }\end{array}$ & $\begin{array}{c}\text { Tumor } \\
\text { incidence }\end{array}$ & $\begin{array}{c}\text { Latency period } \\
\text { (days) }\end{array}$ \\
\hline SKOV3 & $1 \times 10^{3}$ & $0 / 4$ & - \\
& $1 \times 10^{4}$ & $0 / 4$ & - \\
OVCSLC & $1 \times 10^{5}$ & $2 / 4$ & 42 \\
& $1 \times 10^{3}$ & $3 / 4$ & 25 \\
& $1 \times 10^{4}$ & $4 / 4$ & 16 \\
& $1 \times 10^{5}$ & $4 / 4$ & 8 \\
\hline
\end{tabular}

aSKOV3, SKOV3 cells in monolayer growth; OVCSLC, secondgeneration spheroids derived from the SKOV3 cell line.

spheroids, and tumorsphere formation assay via SFM culturing is widely used in their isolation and enrichment in vitro. Under SFM culture conditions, most cancer cells undergo apoptosis, whereas only a small proportion form tumorspheres. These subpopulations of cancer cells are believed to have CSC characteristics such as self-renewal ability and unlimited differentiation. Our group and other investigators have demonstrated that the spheroids of established ovarian cancer cell lines and transplanted human ovarian cancer possess the characteristics of OVCSLCs $(3,4,11-14)$. In the present study, we also identified the characteristics of OVCSLCs in spheroids obtained from SKOV3 cells. We showed that the second-generation spheroids had the highest self-renewal potential (Fig. 1A). We further demonstrated that second-generation spheroids had a higher colony formation rate compared with cells in monolayer growth (Fig. 1B). In addition, the results obtained using western blotting revealed that second-generation spheroids had elevated amounts of CSC-related markers (CD133, CD44 and ALDH1), compared with SKOV3 cells in monolayer growth (Fig. 1C). Significantly, second-generation spheroids displayed more powerful carcinogenicity than cells of the SKOV3 cell line in vivo (Fig. 1D and Table I). These results demonstrated that spheroids derived from the SKOV3 cell line possessed OVCSLC properties, such as higher oncogenicity in vitro and in vivo, and overexpression of 'stemness' biomarkers. For this reason, second-generation spheroids were used as OVCSLCs in the subsequent experimental studies. 
A

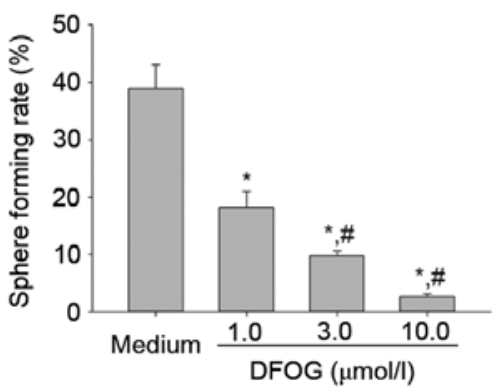

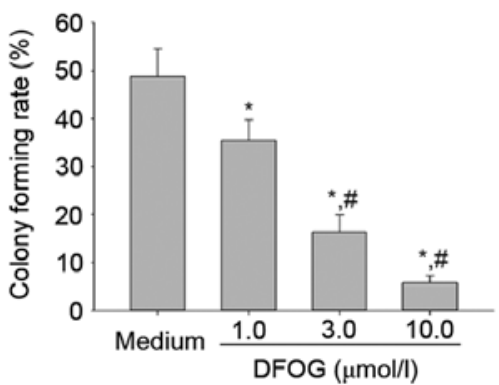

C

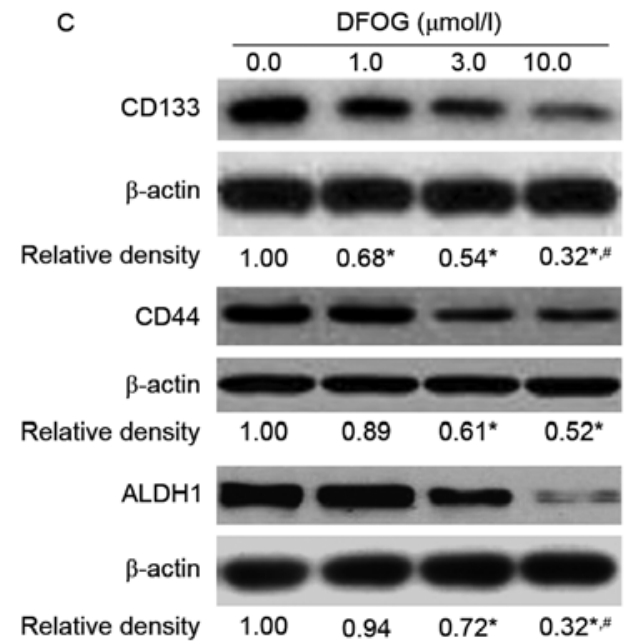

Figure 2. DFOG inhibits the stemness of OVCSLCs derived from SKOV3 cells. DFOG reduced (A) spheroid and (B) colony formation rates, in a concentration-dependent manner (mean $\pm \mathrm{SD}, \mathrm{n}=3$; ${ }^{*} \mathrm{P}<0.05$ compared to the vehicle control group; ${ }^{*} \mathrm{P}<0.05$ compared to $1 \mu \mathrm{mol} / 1 \mathrm{DFOG}$ treatment). (C) DFOG decreased the expression of stem cell markers (mean, $\mathrm{n}=3 ;{ }^{*} \mathrm{P}<0.05$ compared with the vehicle control group; ${ }^{*} \mathrm{P}<0.05$ compared with $1 \mu$ mol/1 $\mathrm{DFOG}$ treatment).

A

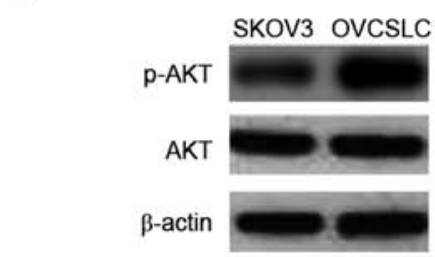

Phosphorylated level $1.00 \quad 3.16^{*}$
B

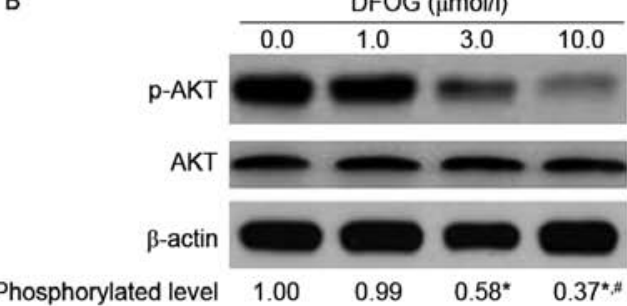

c

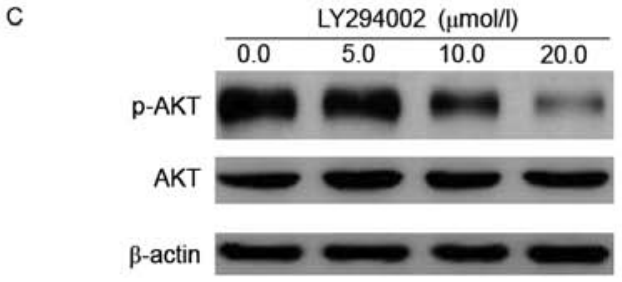

Phosphorylated level $1.00 \quad 0.95 \quad 0.67^{*} \quad 0.38^{* *}$

Figure 3. DFOG inhibits AKT activity of OVCSLCs from SKOV3 cells. (A) Phosphorylated AKT levels in OVCSLCs were higher than those of SKOV3 cells in monolayer growth (mean, $\mathrm{n}=3$; ${ }^{*} \mathrm{P}<0.05$ compared to cells in monolayer growth). (B) DFOG reduced the expression of the p-AKT protein in OVCSLCs from SKOV3 cells (mean, $\mathrm{n}=3$; ${ }^{\text {P }}<0.05$ compared to the vehicle control group; ${ }^{*} \mathrm{P}<0.05$ compared to $1 \mu \mathrm{mol} / 1 \mathrm{DFOG}$ treatment). (C) LY294002 reduced phosphorylation level of AKT in OVCSLCs from SKOV3 cells (mean, $\mathrm{n}=3 ;{ }^{*} \mathrm{P}<0.05$ compared to the vehicle control group; ${ }^{*} \mathrm{P}<0.05 \mathrm{compared}$ to $5.0 \mu$ mol/1 LY294002 treatment).

A

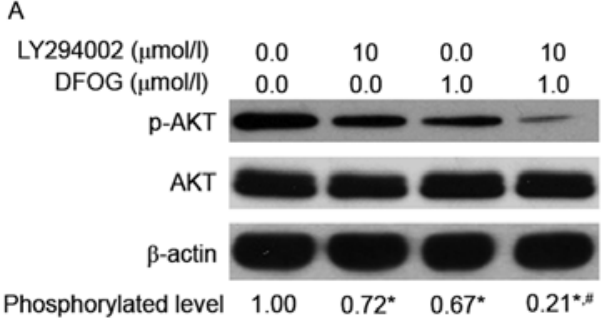

Phosphorylated level $\begin{array}{llll}1.00 & 0.72^{*} & 0.67^{*} & 0.21^{*}, \#\end{array}$
B

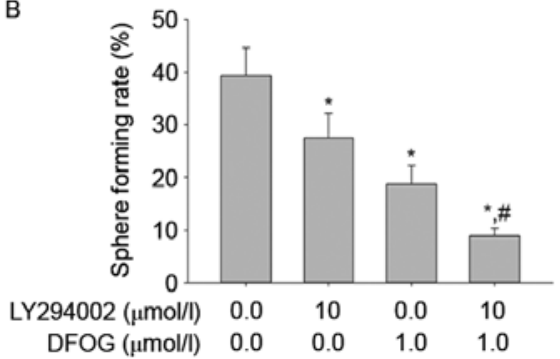

C

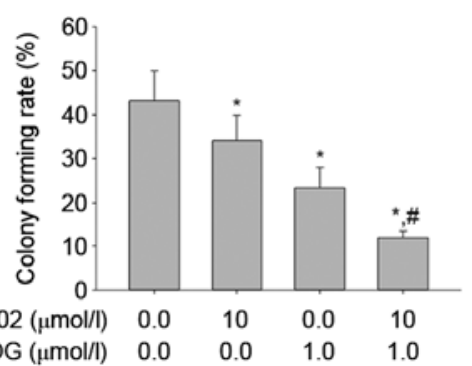

Figure 4. Effects of combined treatment with DFOG and LY294001 on p-AKT protein expression, and spheroid and colony formation in OVCSLCs from SKOV3 cells. (A) Both $10 \mu \mathrm{mol} / 1 \mathrm{LY} 294002$ and $1 \mu \mathrm{mol} / 1$ DFOG decreased the expression of p-AKT, with the latter having a greater inhibitory effect. However, the combination of both inhibitors further decreased $\mathrm{p}$-AKT levels (mean, $\mathrm{n}=3$; ${ }^{*} \mathrm{P}<0.05$ compared to the vehicle control group; ${ }^{*} \mathrm{P}<0.05$ compared to $10 \mu \mathrm{mol} / 1 \mathrm{LY} 294002$ or $1 \mu \mathrm{mol} / 1$ DFOG treatment alone). (B and C) Both $10 \mu \mathrm{mol} / 1 \mathrm{LY} 294002$ and $1 \mu \mathrm{mol} / 1 \mathrm{DFOG}$ reduced (B) spheroid and (C) colony formation rates. Combination of both inhibitors further suppressed spheroid and colony formation (mean $\pm \mathrm{SD}, \mathrm{n}=3$; ${ }^{*} \mathrm{P}<0.05$ compared to the vehicle control group; ${ }^{*} \mathrm{P}<0.05$ compared to $10 \mu \mathrm{mol} / 1 \mathrm{LY} 294002$ or $1 \mu \mathrm{mol} / 1 \mathrm{DFOG}$ treatment alone).

DFOG inhibits the characteristics of SKOV3 cell-produced OVCSLCs. Previous studies revealed the novel genistein analogue DFOG induces apoptosis of various cancer cell lines $(9,15)$. DFOG inhibited the proliferation of CSCs or tumor-initiating cells $(3,4)$. Therefore, we next sought to examine the inhibitory effects of DFOG on stemness of
OVCSLCs from SKOV3 cells. In the present study, we found that DFOG dose-dependently reduced spheroid (Fig. 2A) and colony (Fig. 2B) formation rates. Furthermore, our data provided evidence that DFOG displayed a concentration-dependent downregulation of CSC-related proteins (CD133, CD44 and ALDH1) (Fig. 2C). The current findings suggest that DFOG 
A

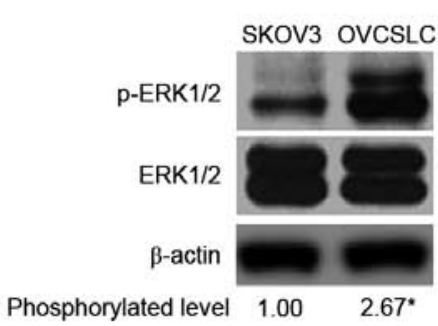

B

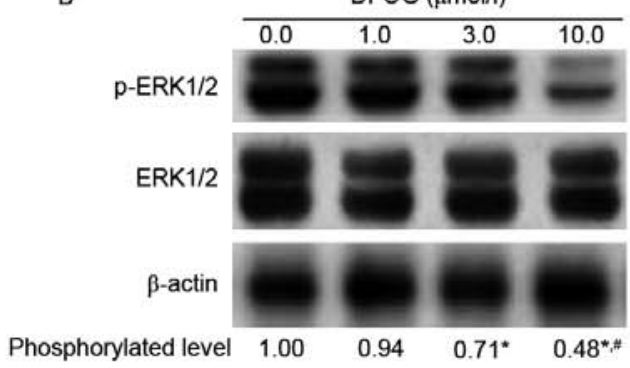

C

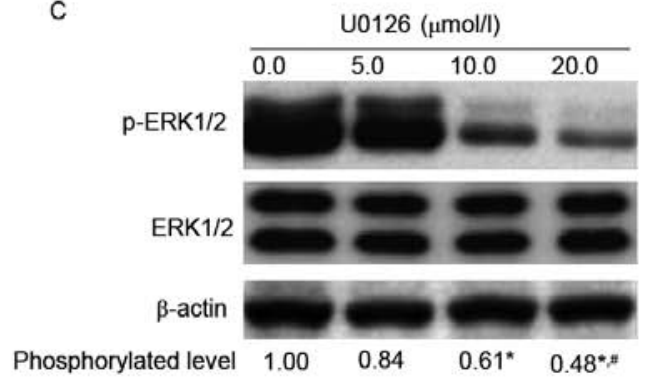

Figure 5. DFOG inhibits ERK1/2 activity of OVCSLCs from SKOV3 cells. (A) The phosphorylation levels of the ERK1/2 protein in OVCSLCs were higher than those of SKOV3 cells in monolayer growth (mean, $\mathrm{n}=3$; ${ }^{*} \mathrm{P}<0.05$ compared with cells in monolayer growth). (B) DFOG reduced p-ERK1/2 protein amounts in OVCSLCs from SKOV3 cells (mean, $\mathrm{n}=3$; ${ }^{*} \mathrm{P}<0.05$ compared to the vehicle control group; ${ }^{\text {}} \mathrm{P}<0.05$ compared to $1 \mu$ mol/ 1 DFOG treatment). (C) U0126 reduced phosphorylation level of ERK1/2 in OVCSLCs from SKOV3 cells (mean, $\mathrm{n}=3$; ${ }^{*} \mathrm{P}<0.05$ compared to the vehicle control group; $\mathrm{P}<0.05$ compared to $5.0 \mu \mathrm{mol} / 1 \mathrm{U} 0126$ treatment).

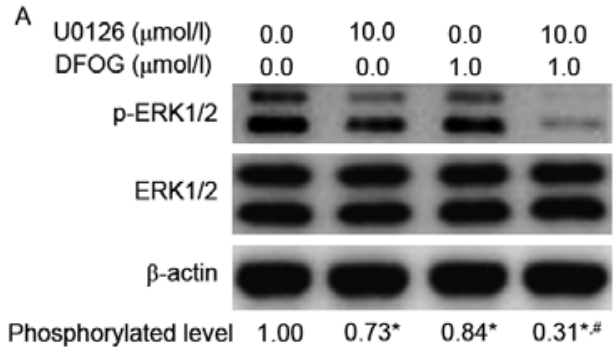

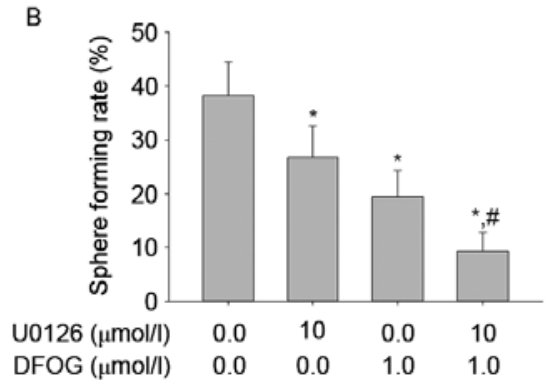

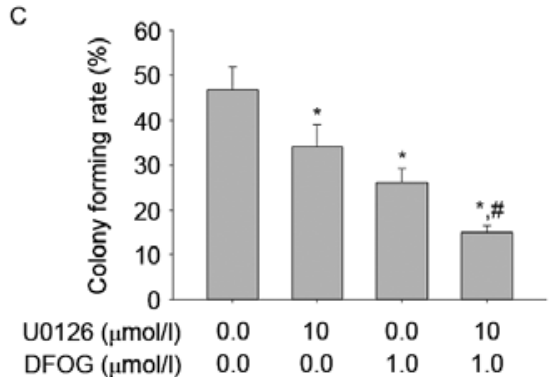

Figure 6. Effects of combined DFOG and U0126 on p-ERK1/2 protein expression, and spheroid and colony formation in OVCSLCs from SKOV3 cells. (A) Both $10 \mu \mathrm{mol} / 1 \mathrm{U} 0126$ and $1 \mu \mathrm{mol} / 1 \mathrm{DFOG}$ decreased p-ERK1/2 levels. Combination of both inhibitors further decreased p-ERK1/2 levels (mean, $\mathrm{n}=3$; ${ }^{*} \mathrm{P}<0.05$ compared to the vehicle control group; ${ }^{\mathrm{P}}<0.05$ compared to $10 \mu \mathrm{mol} / 1 \mathrm{U} 0126$ or $1 \mu \mathrm{mol} / 1 \mathrm{DFOG}$ treatment alone). (B and C) Both $10 \mu \mathrm{mol} / 1 \mathrm{U} 0126$ and $1 \mu \mathrm{mol} / 1$ DFOG reduced (B) spheroid and (C) colony formation rates. Combination of both inhibitors further suppressed spheroid and colony formation (mean $\pm \mathrm{SD}, \mathrm{n}=3 ;{ }^{*} \mathrm{P}<0.05$ compared to the vehicle control group; ${ }^{\#} \mathrm{P}<0.05$ compared to $10 \mu \mathrm{mol} / 1 \mathrm{U} 0126$ or $1 \mu \mathrm{mol} / 1 \mathrm{DFOG}$ treatment alone).

can efficiently inhibit stemness in OVCSLCs from SKOV3 cells.

DFOG reduces the phosphorylation levels of $A K T$ in OVCSLCs from SKOV3 cells. Numerous studies have reported that genistein abolishes stemness in CSCs or CSLCs via inhibition of AKT phosphorylation and activity $(10,16)$. We thus hypothesized that the novel genistein analogue DFOG-inhibited spheroid and colony formation is associated with AKT inactivation. To test the hypothesis, we used western blotting to analyze the protein expression levels of p-AKT in cells treated with DFOG or not. As expected, elevated amounts of phosphorylated AKT in OVCSLCs were obtained compared with the levels in SKOV3 cells grown in monolayers (Fig. 3A). DFOG effects (Fig. 3B) were similar to those of the PI3K inhibitor LY294002 (Fig. 3C), effectively and concentration-dependently reducing the phosphorylation levels of the AKT protein in SKOV3 cell-produced OVCSLCs.

To confirm that the reduced AKT phosphorylation is involved in oncogenicity maintenance in vitro in SKOV3 cell-produced OVCSLCs, we next sought to examine p-AKT levels, and spheroid and colony forming capabilities in OVCSLCs treated with DFOG $(1 \mu \mathrm{mol} / \mathrm{l})$ and/or LY294002 (10 $\mu \mathrm{mol} / \mathrm{l})$. We found that DFOG and LY294002 cooperated to reduce the levels of AKT phosphorylation (Fig. 4A), and attenuate spheroid (Fig. 4B) and colony (Fig. 4C) forming capabilities in OVCSLCs from the SKOV3 cell line. These results suggest that DFOG-inhibited spheroid and colony formation may be associated with AKT inactivation in OVCSLCs from SKOV3 cells.

DFOG downregulates p-ERK1/2 expression in OVCSLCs from SKOV3 cells. ERK kinase signaling is essential for cell proliferation. In healthy cells, the activated signaling pathway leads to progression from $\mathrm{G} 1$ to $\mathrm{S}$ phase, and is involved in the inactivation of antiproliferative genes. Moreover, inhibition of the ERK pathway reduces the development of CSCs (17). Increasing evidence demonstrates that genistein inhibits self-renewal ability and CSC-related protein expression in various CSCs or CSLCs via inhibition of ERK1/2 phosphorylation (18-21). We next sought to analyze whether DFOG-inhibited spheroid and colony formation is associated with reduced ERK1/2 protein phosphorylation. We found elevated levels of phosphorylated ERK in OVCSLCs compared with the amounts obtained for SKOV3 cells in monolayer growth (Fig. 5A). DFOG (Fig. 5B) showed similar effects to the MEK inhibitor U0126 (Fig. 5C), effectively concentration-dependently reducing the phosphorylation levels of the ERK1/2 protein in OVCSLCs derived from SKOV3 cells.

To determine how DFOG-related reduced ERK1/2 protein phosphorylation affects oncogenicity maintenance in vitro in SKOV3 cell-produced OVCSLCs, we next sought to examine 
A

B

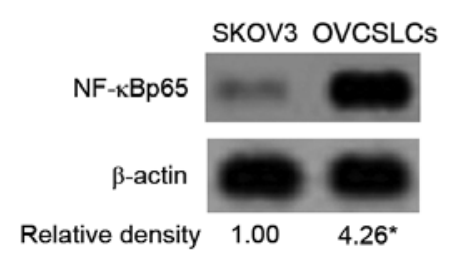

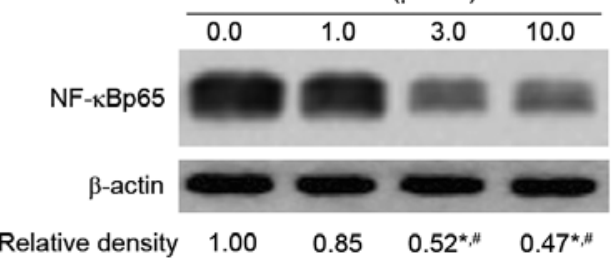

C

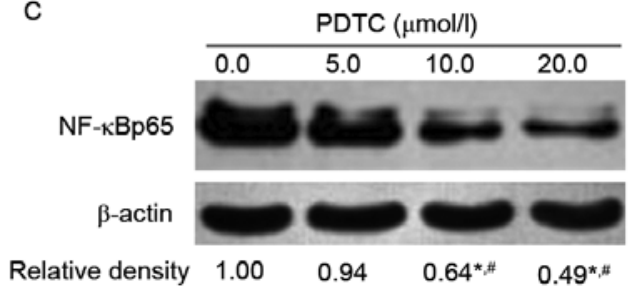

Figure 7 DFOG inhibits NF- $\mathrm{B}$ activity in OVCSLCs from SKOV3 cells. (A) The protein expression levels of NF- $\mathrm{kp} 65$ in OVCSLCs were higher than those of SKOV3 cells in monolayer growth (mean, $\mathrm{n}=3$; ${ }^{*} \mathrm{P}<0.05$ compared to cells in monolayer growth). (B) DFOG inhibited the protein expression ofNF- $\mathrm{kBp} 65$ in OVCSLCs from SKOV3 cells (mean, $\mathrm{n}=3$; ${ }^{*} \mathrm{P}<0.05$ compared to the vehicle control group; ${ }^{\text {}} \mathrm{P}<0.05$ compared to $1 \mu$ mol/ 1 DFOG treatment). (C) PDTC decreased NF- $\kappa$ Bp 65 protein levels in OVCSLCs from SKOV3 cells (mean, $\mathrm{n}=3 ;{ }^{*} \mathrm{P}<0.05$ compared to the vehicle control group; ${ }^{\#} \mathrm{P}<0.05$ compared to $5.0 \mu \mathrm{mol} / 1$ PDTC treatment).

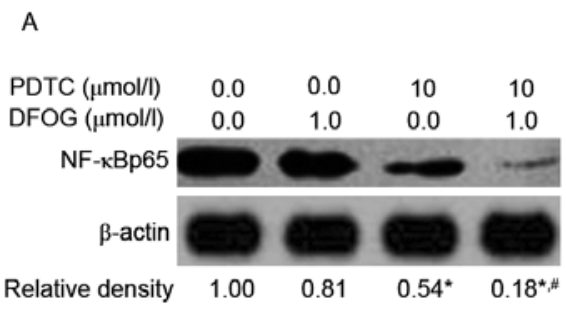

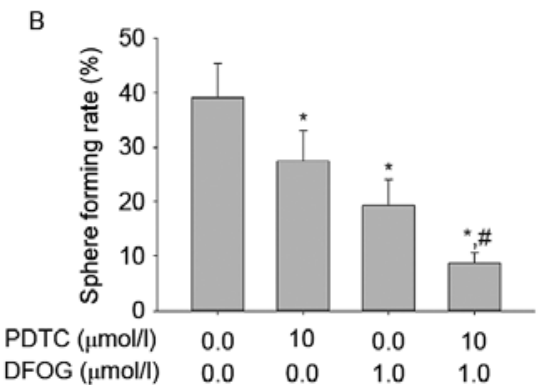

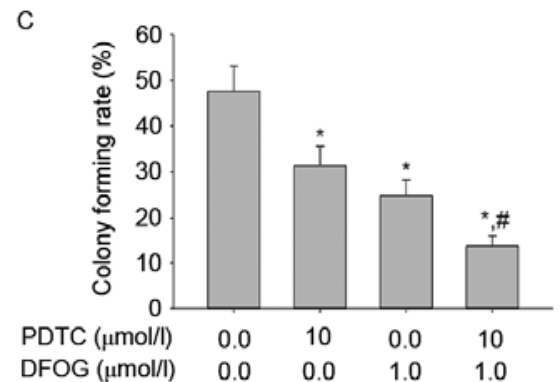

Figure 8. Effects of combined DFOG and PDTC on NF- $\mathrm{Bp} 65$ expression, and spheroid and colony formation in OVCSLCs from SKOV3 cells. (A) Both $10 \mu$ mol/1 PDTC and $1 \mu \mathrm{mol} / 1$ DFOG decreased the expression of NF- $\kappa$ Bp65. Combination of both inhibitors further suppressed the expression of NF- $\kappa$ Bp65 (mean, $\mathrm{n}=3$; ${ }^{*} \mathrm{P}<0.05$ compared to the vehicle control group; ${ }^{\#} \mathrm{P}<0.05$ compared to $10 \mu \mathrm{mol} / 1 \mathrm{PDTC}$ or $1 \mu \mathrm{mol} / 1 \mathrm{DFOG}$ treatment alone). (B and C) Both $10 \mu \mathrm{mol} / 1 \mathrm{PDTC}$ and $1 \mu \mathrm{mol} / 1$ DFOG reduced (B) spheroid and (C) colony formation rates. Combination of both inhibitors further suppressed spheroid formation and colony formation (mean, $\mathrm{n}=3 ;{ }^{*} \mathrm{P}<0.05$ compared to the vehicle control group; ${ }^{\text {}} \mathrm{P}<0.05$ compared to $10 \mu \mathrm{mol} / 1 \mathrm{PDTC}$ or $1 \mu \mathrm{mol} / 1 \mathrm{DFOG}$ treatment alone).

p-ERK levels, spheroid and colony forming capabilities in OVCSLCs treated with DFOG $(1 \mu \mathrm{mol} / \mathrm{l})$ and/or U0126 (10 $\mu \mathrm{mol} / \mathrm{l})$. We showed that DFOG and U0126 cooperated to reduce the levels of ERK1/2 protein phosphorylation (Fig. 6A), and attenuate spheroid (Fig. 6B) and colony (Fig. 6C) forming capabilities in OVCSLCs from the SKOV3 cell line. These results suggest that DFOG-inhibited spheroid and colony formation may be associated with inhibition of ERK1/2 protein phosphorylation in OVCSLCs from SKOV3 cells.

DFOG decreases $N F-\kappa B$ activity in OVCSLCs from SKOV3 cells. Recent studies showed that genistein and its derivatives inhibit the proliferation and invasion of various cancer cells or cancer stem-like cells by inhibiting NF- $\kappa \mathrm{B}$ nuclear translocation via IкB signaling $(19,22,23)$. We next sought to assess whether DFOG-inhibited spheroid and colony formation is associated with the inhibition of NF- $\kappa \mathrm{B}$ activity. The results obtained by western blot analysis demonstrated an upregulation of $\mathrm{NF}-\kappa \mathrm{Bp} 65$, an active fragment of $\mathrm{NF}-\kappa \mathrm{B}$, in OVCSLCs compared with cells in monolayer growth derived from the SKOV3 cell line (Fig. 7A). Both DFOG (Fig. 7B) and PDTC (Fig. 7C), a NF- $\kappa$ B inhibitor, dose-dependently reduced the expression levels of NF- $\kappa \mathrm{Bp} 65$.

To examine the role of DFOG-related inhibition of $\mathrm{NF}-\kappa \mathrm{B}$ activity in oncogenicity maintenance in vitro in SKOV3 cell-produced OVCSLCs, we next sought to quantify NF-кBp65 levels, and spheroid and colony forming capabilities in OVCSLCs treated with DFOG $(1 \mu \mathrm{mol} / \mathrm{l})$ or PDTC
(10 $\mu \mathrm{mol} / \mathrm{l})$ or both. We found that DFOG and PDTC cooperated to reduce the levels of NF- $\kappa \mathrm{Bp} 65$ expression (Fig. 8A), and attenuate spheroid (Fig. 8B) and colony (Fig. 8C) forming capabilities in OVCSLCs from the SKOV3 cell line. These results suggest that DFOG-inhibited spheroid and colony formation may be associated with the inhibition of $N F-\kappa B$ activity in OVCSLCs from SKOV3 cells.

Inhibitory effects of DFOG on oncogenicity in vitro depend on FoxO3a expression in OVCSLCs from SKOV3 cells. Since the FoxO3a function is closely associated with OVCSLC oncogenicity inhibition related to suppressed AKT and/or ERK and/or NF- $\kappa$ B pathway $(1,24,25)$, whether oncogenicity reduction by DFOG in vitro depended on FoxO3a expression in OVCSLCs from SKOV3 cells was assessed. Fig. 9A shows elevated expression levels of phosphorylated FoxO3a in OVCSLCs compared with these levels in SKOV3 cells in monolayer growth. DFOG (Fig. 9B) effectively and concentration-dependently reduced the phosphorylation levels of FoxO3a in SKOV3 cell-produced OVCSLCs. In SKOV3 cell-produced OVCSLCs, FoxO3a expression was knocked down by transduction with the FOXO3a shRNA-expressing adenovirus (Fig. 9C). Our results revealed that co-treatment with DFOG and FOXO3a shRNA reduced the expression levels of FoxO3a (Fig. 9D), and synergistically attenuated the spheroid (Fig. 9E) and colony (Fig. 9F) forming capabilities of OVCSLCs from the SKOV3 cell line. Taken together, these data indicate that inhibition of OVCSLC oncogenicity 
A

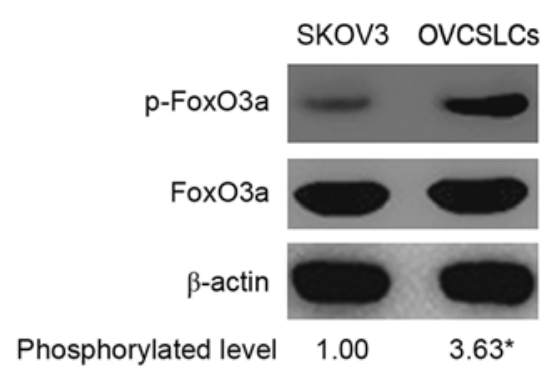

C

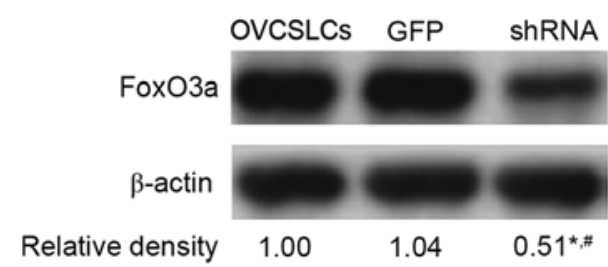

E

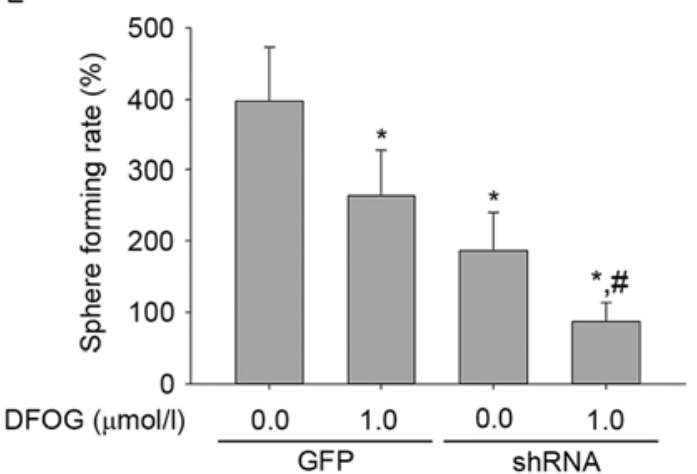

B

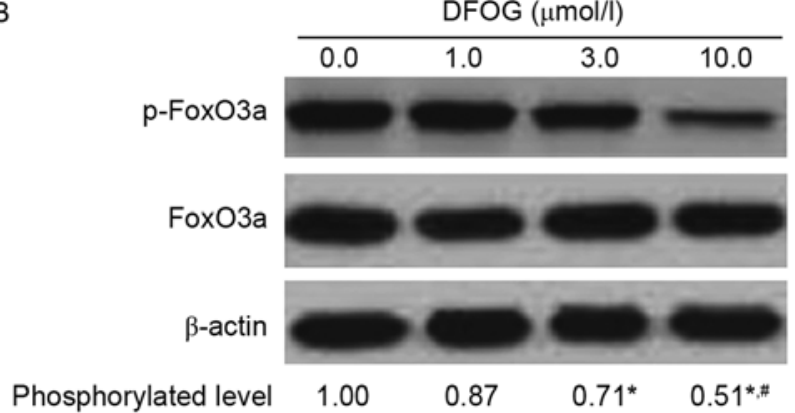

D

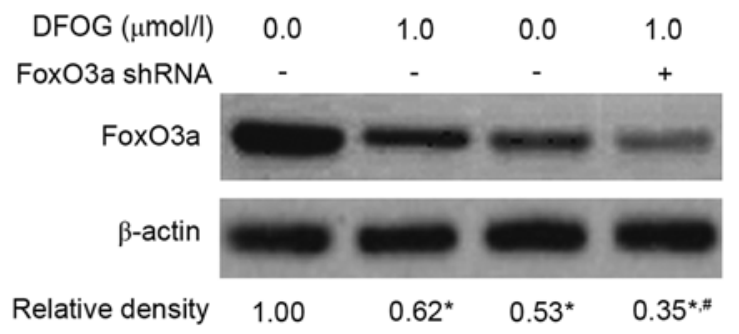

F

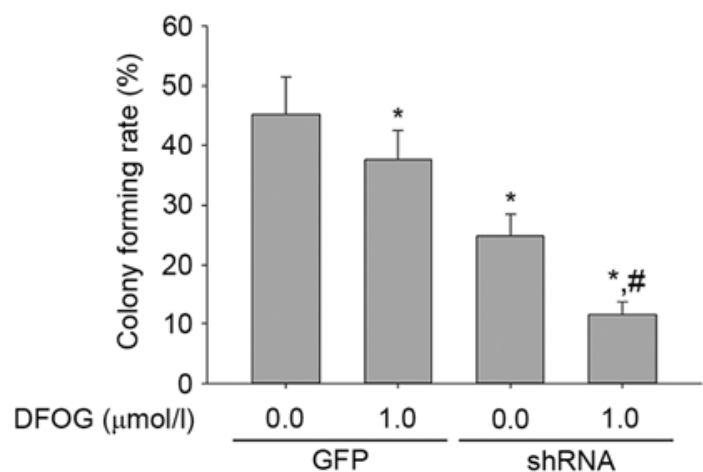

Figure 9. Effects of combined DFOG and FoxO3a shRNA on FoxO3a expression, and spheroid and colony formation in OVCSLCs from SKOV3 cells. (A) Comparison of protein expression of $\mathrm{p}$-FoxO3a in OVCSLCs (second-generation spheroids) and SKOV3 cells in monolayer growth (mean, $\mathrm{n}=3$; ${ }^{*} \mathrm{P}<0.05$ compared to cells in monolayer growth). (B) DFOG inhibited the protein expression of $\mathrm{p}-\mathrm{FoxO} \mathrm{a}$, in a concentration-dependent manner (mean, $\mathrm{n}=3$; ${ }^{*} \mathrm{P}<0.05$ compared to the vehicle control group; ${ }^{\text {P }}<0.05$ compared to $1 \mu \mathrm{mol} / 1$ DFOG treatment). (C) FOXO3a gene knockdown decreased the protein expression of FoxO3a (mean, $\mathrm{n}=3 ;{ }^{*} \mathrm{P}<0.05$ compared to SKOV3 cell-produced OVCSLCs; ${ }^{\text {P }}<0.05$ compared to OVCSLCs transduced with adenovirus containing the GFP gene). (D) FOXO3a shRNA reduced the expression levels of FoxO3a; Combination of DFOG and FOXO3a shRNA further suppressed the expression of FoxO3a (mean, $\mathrm{n}=3 ;{ }^{*} \mathrm{P}<0.05$ compared to untreated SKOV3 cell-produced OVCSLCs; ${ }^{*} \mathrm{P}<0.05$ compared to FOXO3a shRNA or $1 \mu$ mol/1 DFOG treatment alone). (E and F) Both FOXO3a shRNA and $1 \mu \mathrm{mol} / 1$ DFOG reduced (E) spheroid and (F) colony formation rates. Combination of FOXO3a shRNA and $1 \mu \mathrm{mol} / 1 \mathrm{DFOG}$ further suppressed spheroid and colony formation (mean $\pm \mathrm{SD}, \mathrm{n}=3$; ${ }^{*} \mathrm{P}<0.05$ compared to untreated SKOV3 cell-produced $\mathrm{OVCSLCs}$; ${ }^{\#} \mathrm{P}<0.05$ compared to FOXO3a shRNA and $1 \mu \mathrm{mol} / 1$ DFOG treatment alone).

in vitro mediated by the suppressive effects of DFOG on $\mathrm{AKT}$ and/or ERK and/or $\mathrm{NF}-\kappa \mathrm{B}$ pathways requires FoxO3a expression.

Oncogenicity reduction by DFOG in vitro requires FoxMI in OVCSLCs from SKOV3 cells. A study by McGovern et al demonstrated ectopically expressed FOXO3a downregulates FOXM1, while FOXO3a silencing increases FOXM1 levels and rescues sensitive breast cancer BT474 cells from gefitinib-related growth inhibition (26). Our results suggested that FoxM1 inhibition by FoxO3a activation induced apoptosis in ovarian cancer cells (27). Furthermore, previous studies demonstrated that FoxM1 inhibition results in altered characteristics of OVCSLCs from SKOV3 cells (4). Importantly, Bao et al found that genistein inhibits cell malignancy, in agreement with decreased CD44 and EpCAM levels. Accordingly, we determined that the inhibitory effects of DFOG on onco- genicity in vitro depended on FoxM1 expression in OVCSLCs from $\mathrm{SKOV} 3$ cells.

Fig. 10A shows FoxM1 upregulation in OVCSLCs compared with the levels of SKOV3 cells in monolayer growth. DFOG (Fig. 10B) effectively and concentration-dependently reduced the expression of the FoxM1 protein in OVCSLCs derived from SKOV3 cells. In SKOV3 cell-produced OVCSLCs, FoxM1 expression was knocked down by transduction with FoxM1 shRNA-expressing adenovirus (Fig. 10C). Our results also revealed that co-treatment with DFOG and FOXM1 shRNA reduced the expression levels of FoxM1 (Fig. 10D), and synergistically attenuated spheroid (Fig. 10E) and colony (Fig. 10F) forming capabilities in OVCSLCs from the SKOV3 cell line. Taken together, these data indicate that reduced OVCSLC oncogenicity in vitro by DFOG through inhibitory effects on AKT and/or ERK and/or NF- $\kappa \mathrm{B}$ pathways requires FoxM1 expression. 
A

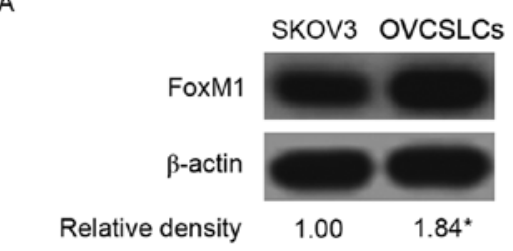

C

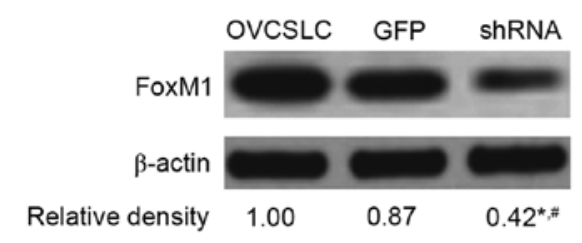

E

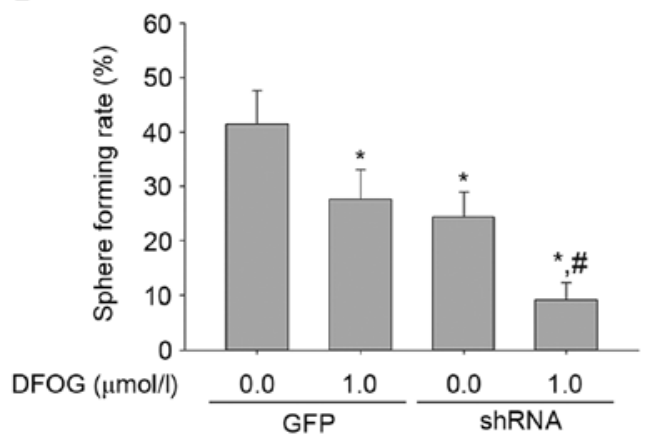

B

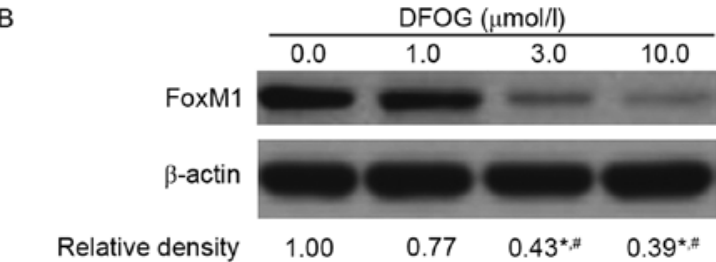

D
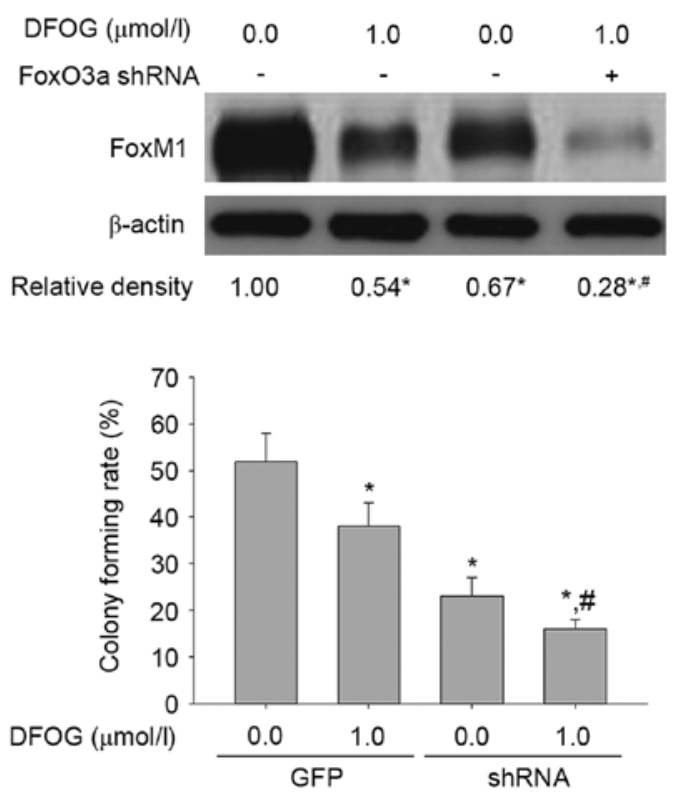

Figure 10. Effects of combined of DFOG and FoxM1 shRNA on FoxM1 expression, and spheroid and colony formation in OVCSLCs from SKOV3 cells. (A) Comparison of protein expression of FoxM1 in OVCSLCs (second-generation spheroids) and SKOV3 cells in monolayer growth (mean, $\mathrm{n}=3$; ${ }^{*} \mathrm{P}<0.05$ compared to cells in monolayer growth). (B) DFOG concentration-dependently reduced FoxM1 protein amounts (mean, $\mathrm{n}=3$; ${ }^{*} \mathrm{P}<0.05$ compared to the vehicle control group; " $\mathrm{P}<0.05$ compared to $1 \mu \mathrm{mol} / 1 \mathrm{DFOG}$ treatment). (C) FOXM1 gene knockdown decreased expression of FoxM1 protein (mean, $\mathrm{n}=3$; ${ }^{*} \mathrm{P}<0.05$ compared to SKOV3 cell-produced OVCSLCs; ${ }^{\#} \mathrm{P}<0.05$ compared to OVCSLCs transduced with adenovirus containing the GFP gene). (D) FOXM1 shRNA reduced the expression levels of FoxM1. Combination of DFOG and FOXM1 shRNA further suppressed the expression of FoxM1 protein (mean, $\mathrm{n}=3$; ${ }^{*} \mathrm{P}<0.05$ compared to untreated SKOV3 cell produced OVCSLCs; ${ }^{P} \mathrm{P}<0.05$ compared to FOXO3a shRNA or $1 \mu \mathrm{mol} / 1$ DFOG treatment alone). (E and F) Both FOXM1 shRNA and $1 \mu \mathrm{mol} / 1$ DFOG reduced (E) spheroid and (F) colony formation rates. Combination of FOXM1 shRNA and $1 \mu$ mol/1 DFOG further suppressed spheroid and colony formation (mean $\pm \mathrm{SD}, \mathrm{n}=3$; ${ }^{*} \mathrm{P}<0.05$ compared to untreated SKOV3 cell produced OVCSLCs; ${ }^{*} \mathrm{P}<0.05$ vs. FOXM1 shRNA and $1 \mu$ mol/1 DFOG treatment alone).

\section{Discussion}

Cancer is the uncontrolled growth of cells. Numerous signaling pathways regulate cell growth and proliferation (28). Various molecules are associated with tumor development. For example, phosphorylation of AKT, activation of ERK1 and ERK2, and NF- $\kappa$ Bp65 proteins lead to cancer cell development (24). In the present study, we ascertained whether a newly synthesized potential anticancer genistein analogue, DFOG, possesses inhibitory effects on p-AKT and $\mathrm{p}-\mathrm{ERK} \mathrm{K} 1 / 2$ expression, and $\mathrm{NF}-\kappa \mathrm{B}$ activity, as well as on the suppression of oncogenicity in vitro in SKOV3-derived OVCSLCs. SKOV3-derived spheroids were therefore used to demonstrate the pharmacological effectiveness of the inhibitors. The other drugs, including LY294002, U0126 and PDTC, whose chemopreventive activities have been demonstrated in previous studies, were compared to DFOG (29). Notably, in the present study, we found that DFOG significantly reduced the levels of phosphorylated AKT. Combination of DFOG with another pharmacological inhibitor LY294002 led to a drastic decrease in $\mathrm{p}$-AKT expression. In addition, the target drug was capable of downregulating phosphorylated ERK1 and
ERK2 proteins in the ERK1/2 signaling pathway. However, DFOG compared with another tumor inhibitor (U0126) was likely to be less effective in diminishing the activation of key proteins involved in cell growth and survival. Nevertheless, simultaneous application of the two anticancer agents resulted in a significant inhibition of phosphorylated ERK1 and ERK2 levels. Finally, the genistein analogue DFOG demonstrated a high potential to decrease the level of the NF- $\kappa$ Bp65 protein, which is extremely important in chemopreventive therapies against cancer development. However, based on its antitumor potency, the novel genistein derivative DFOG is considered an agent with activity against different types of cancer (30).

The present study revealed the anticancer activity of DFOG in OVCSLCs. Cancer stem cells (CSCs) are known to maintain and facilitate the formation of cancers. In addition, CSLCs drive drug resistance as well as recurrence or relapse (31). As ovarian cancer is resistant to conventional chemotherapies, a new approach to target CSCs is necessary. Compounds containing genistein can be applied against CSCs (32). Thus, in the present study, we determined how an anticancer analogue of genistein, DFOG, affects OVCSLCs. A study by Roy et al (33) demonstrated that repressing PI3K/AKT and MEK/ERK pathways 
induces FOXO transcription factors. After transduction with the FOXO3a shRNA-expressing adenovirus, we found that the combination of DFOG and FOXO3a shRNA downregulated FoxO3a, synergistically attenuating spheroid and colony forming capabilities of SKOV3 cell-produced OVCSLCs. To integrate the abovementioned tight association of FoxO3a with OVCSLC oncogenicity inhibition related to AKT and/or ERK and/or NF- $\kappa \mathrm{B}$ pathway suppression, our data showed that reduced OVCSLC oncogenicity in vitro due to the suppressive effects of DFOG on AKT and/or ERK and/or NF- $\kappa$ B pathways requires FoxO3a expression. These results support the viewpoint of Sunayama et al (1) that FoxO3a likely has an important function in controlling CSLCs via PI3K/AKT/mTOR and MEK/ERK signaling, thereby implying that tools effectively targeting FoxO3a induction may constitute a viable option for human carcinoma treatment.

McGovern et al (26) assessed FOXM1 function and modulation after gefitinib treatment, and demonstrated that gefitinib downregulates FOXM1 through FOXO3a in breast carcinoma. Our previous study demonstrated that DFOG acts as an inhibitor of CSCs or tumor-initiating cells by activating FoxO3a and/ or inactivating FoxM1 (4). As demonstrated above, combining DFOG and FOXM1 shRNA reduced the expression levels of FoxM1, and synergistically attenuated the spheroid and colony forming capabilities of OVCSLCs from the SKOV3 cell line. These results indicated that reduced OVCSLC oncogenicity in vitro by DFOG through inhibitory effects on AKT and/or ERK and/or NF- $\kappa \mathrm{B}$ pathways requires FoxM1 expression.

Overall, DFOG exerts anticancer activity by targeting OVCSLCs. DFOG may be effective in reducing the expression levels of cell regulatory proteins such as p-AKT, p-ERK1/2, and $\mathrm{NF}-\kappa \mathrm{Bp} 65$. Moreover, the novel genistein derivative prevented spheroid and colony formation in OVCSLCs. These findings provide evidence for DFOG potency in the inhibition of cancer progression. Importantly, the reduced OVCSLC oncogenicity in vitro by DFOG through inhibitory effects on $\mathrm{AKT}$ and/or ERK and/or NF- $\mathrm{BB}$ pathways requires both FoxO3a and FoxM1 expression. Therefore, DFOG may be used as a novel chemotherapeutic drug for ovarian carcinoma.

\section{Acknowledgements}

The present study was funded by the National Natural Science Foundation of China (nos. 81301894 and 81302249), the Guangzhou Science and Information Bureau Item (no. 201300000151), the Guangdong Province Department of Science and Technology of China (nos. 2014A020211028, 2014A020212609 and 2012B031800271) and the Scientific Research Project for Medical College of Bureau of Education of Guangzhou City (no. 1201410508).

\section{References}

1. Sunayama J, Sato A, Matsuda K, Tachibana K, Watanabe E, Seino S, Suzuki K, Narita Y, Shibui S, Sakurada K, et al: FoxO3a functions as a key integrator of cellular signals that control glioblastoma stem-like cell differentiation and tumorigenicity. Stem Cells 29: 1327-1337, 2011.

2. Jacobsen EA, Ananieva O, Brown $\mathrm{ml}$ and Chang Y: Growth, differentiation, and malignant transformation of pre-B cells mediated by inducible activation of $\mathrm{v}$-Abl oncogene. J Immunol 176: 6831-6838, 2006.
3. Ning Y, Luo C, Ren K, Quan M and Cao J: FOXO3a-mediated suppression of the self-renewal capacity of sphere-forming cells derived from the ovarian cancer SKOV3 cell line by 7-difluoromethoxyl-5,4'-di-n-octyl genistein. Mol Med Rep 9: 1982-1988, 2014.

4. Ning YX, Li QX, Ren KQ, Quan MF and Cao JG: 7-difluoromethoxyl-5,4'-di-n-octyl genistein inhibits ovarian cancer stem cell characteristics through the downregulation of FOXM1. Oncol Lett 8: 295-300, 2014.

5. Li Y, Wicha MS, Schwartz SJ and Sun D: Implications of cancer stem cell theory for cancer chemoprevention by natural dietary compounds. J Nutr Biochem 22: 799-806, 2011.

6. Oh J, Hlatky L, Jeong YS and Kim D: Therapeutic effectiveness of anticancer phytochemicals on cancer stem cells. Toxins 8: pii: E199, 2016.

7. Cunha-Rodrigues M, Portugal S, Prudêncio M, Gonçalves LA, Casalou C, Buger D, Sauerwein R, Haas W and Mota MM: Genistein-supplemented diet decreases malaria liver infection in mice and constitutes a potential prophylactic strategy. PLoS One 3: e2732, 2008.

8. Zhou HB, Chen JM, Cai JT, Du Q and Wu CN: Anticancer activity of genistein on implanted tumor of human SG7901 cells in nude mice. World J Gastroenterol 14: 627-631, 2008.

9. Ning Y, Li Q, Xiang H, Liu F and Cao J: Apoptosis induced by 7-difluoromethoxyl-5,4'-di-n-octyl genistein via the inactivation of FoxM1 in ovarian cancer cells. Oncol Rep 27: 1857-1864, 2012.

10. Montales MT, Rahal OM, Kang J, Rogers TJ, Prior RL, Wu X and Simmen RC: Repression of mammosphere formation of human breast cancer cells by soy isoflavone genistein and blueberry polyphenolic acids suggests diet-mediated targeting of cancer stem-like/progenitor cells. Carcinogenesis 33: 652-660, 2012.

11. Chung H, Kim YH, Kwon M, Shin SJ, Kwon SH, Cha SD and Cho CH: The effect of salinomycin on ovarian cancer stem-like cells. Obstet Gynecol Sci 59: 261-268, 2016.

12. Latifi A, Luwor RB, Bilandzic M, Nazaretian S, Stenvers K, Pyman J, Zhu H, Thompson EW, Quinn MA, Findlay JK, et al: Isolation and characterization of tumor cells from the ascites of ovarian cancer patients: Molecular phenotype of chemoresistant ovarian tumors. PLoS One 7: e46858, 2012.

13. Vermeersch KA, Wang L, Mezencev R, McDonald JF and Styczynski MP: OVCAR-3 spheroid-derived cells display distinct metabolic profiles. PLoS One 10: e0118262, 2015.

14. Condello S, Morgan CA, Nagdas S, Cao L, Turek J, Hurley TD and Matei D: $\beta$-Catenin-regulated ALDH1A1 is a target in ovarian cancer spheroids. Oncogene 34: 2297-2308, 2015.

15. Xiang HL, Liu F, Quan MF, Cao JG and Lv Y: 7-difluoromethoxyl-5,4'-di-n-octylgenistein inhibits growth of gastric cancer cells through downregulating forkhead box M1. World J Gastroenterol 18: 4618-4626, 2012.

16. Liu Y, Zou T, Wang S, Chen H, Su D, Fu X, Zhang Q and Kang X: Genistein-induced differentiation of breast cancer stem/progenitor cells through a paracrine mechanism. Int J Oncol 48: 1063-1072, 2016.

17. Yao Y, Li W, Wu J, Germann UA, Su MS, Kuida K and Boucher DM: Extracellular signal-regulated kinase 2 is necessary for mesoderm differentiation. Proc Natl Acad Sci USA 100: 12759-12764, 2003.

18. Huang W, Wan C, Luo Q, Huang Z and Luo Q: Genistein-inhibited cancer stem cell-like properties and reduced chemoresistance of gastric cancer. Int J Mol Sci 15: 3432-3443, 2014.

19. Wang SD, Chen BC, Kao ST, Liu CJ and Yeh CC: Genistein inhibits tumor invasion by suppressing multiple signal transduction pathways in human hepatocellular carcinoma cells. BMC Complement Altern Med 14: 26, 2014.

20. Kim SH, Kim SH, Kim YB, Jeon YT, Lee SC and Song YS: Genistein inhibits cell growth by modulating various mitogen-activated protein kinases and AKT in cervical cancer cells. Ann NY Acad Sci 1171: 495-500, 2009.

21. de Blas E, Estañ MC, Del Carmen Gómez de Frutos M, Ramos J, Del Carmen Boyano-Adánez M and Aller P: Selected polyphenols potentiate the apoptotic efficacy of glycolytic inhibitors in human acute myeloid leukemia cell lines. Regulation by protein kinase activities. Cancer Cell Int 16: 70, 2016.

22. Wang Y, Lu P, Zhang W, Du Q, Tang J, Wang H, Lu J and Hu R: GEN-27, a newly synthetic isoflavonoid, inhibits the proliferation of colon cancer cells in inflammation microenvironment by suppressing NF- $\kappa$ B pathway. Mediators Inflamm 2016: 2853040, 2016. 
23. Vazquez-Santillan K, Melendez-Zajgla J, Jimenez-Hernandez LE, Gaytan-Cervantes J, Muñoz-Galindo L, Piña-Sanchez P, Martinez-Ruiz G, Torres J, Garcia-Lopez P, Gonzalez-Torres C, et al: NF-kappaB-inducing kinase regulates stem cell phenotype in breast cancer. Sci Rep 6: 37340, 2016.

24. Jung Y, Park H, Zhao HY, Jeon R, Ryu JH and Kim WY: Systemic approaches identify a garlic-derived chemical, Z-ajoene, as a glioblastoma multiforme cancer stem cell-specific targeting agent. Mol Cells 37: 547-553, 2014.

25. Dubrovska A, Kim S, Salamone RJ, Walker JR, Maira SM, García-Echeverría C, Schultz PG and Reddy VA: The role of PTEN/Akt/PI3K signaling in the maintenance and viability of prostate cancer stem-like cell populations. Proc Natl Acad Sci USA 106: 268-273, 2009.

26. McGovern UB, Francis RE, Peck B, Guest SK, Wang J, Myatt SS, Krol J, Kwok JM, Polychronis A, Coombes RC, et al: Gefitinib (Iressa) represses FOXM1 expression via FOXO3a in breast cancer. Mol Cancer Ther 8: 582-591, 2009.

27. Jiang L, Cao XC, Cao JG, Liu F, Quan MF, Sheng XF and Ren KQ: Casticin induces ovarian cancer cell apoptosis by repressing FoxM1 through the activation of FOXO3a. Oncol Lett 5: 1605-1610, 2013.
28. Steelman LS, Chappell WH, Abrams SL, Kempf RC, Long J, Laidler P, Mijatovic S, Maksimovic-Ivanic D, Stivala F, Mazzarino MC, et al: Roles of the Raf/MEK/ERK and $\mathrm{PI} 3 \mathrm{~K} / \mathrm{PTEN} / \mathrm{Akt} / \mathrm{mTOR}$ pathways in controlling growth and sensitivity to therapy-implications for cancer and aging. Aging 3: 192-222, 2011.

29. Smalley KS, Haass NK, Brafford PA, Lioni M, Flaherty KT and Herlyn M: Multiple signaling pathways must be targeted to overcome drug resistance in cell lines derived from melanoma metastases. Mol Cancer Ther 5: 1136-1144, 2006.

30. Chahar MK, Sharma N, Dobhal MP and Joshi YC: Flavonoids: A versatile source of anticancer drugs. Pharmacogn Rev 5: 1-12, 2011.

31. Sakariassen PO, Immervoll $\mathrm{H}$ and Chekenya M: Cancer stem cells as mediators of treatment resistance in brain tumors: Status and controversies. Neoplasia 9: 882-892, 2007.

32. Zhang L, Li L, Jiao M, Wu D, Wu K, Li X, Zhu G, Yang L, Wang X, Hsieh JT, et al: Genistein inhibits the stemness properties of prostate cancer cells through targeting Hedgehog-Gli1 pathway. Cancer Lett 323: 48-57, 2012.

33. Roy SK, Srivastava RK and Shankar S: Inhibition of PI3K/AKT and MAPK/ERK pathways causes activation of FOXO transcription factor, leading to cell cycle arrest and apoptosis in pancreatic cancer. J Mol Signal 5: 10, 2010. 